Archives

43 | 2009

Hommage à Jacques Ozouf

\title{
Lire et écrire
}

Une enquête, un moment historiographique

Dominique Julia et Jacques Revel

\section{(2) OpenEdition}

Journals

Édition électronique

URL : https://journals.openedition.org/ccrh/3497

DOI : $10.4000 /$ ccrh.3497

ISSN : $1760-7906$

Éditeur

Centre de recherches historiques - EHESS

Édition imprimée

Date de publication : 25 janvier 2009

Pagination : 35-56

ISSN : 0990-9141

Référence électronique

Dominique Julia et Jacques Revel, «Lire et écrire », Les Cahiers du Centre de Recherches Historiques [En ligne], 43 | 2009, mis en ligne le 16 février 2012, consulté le 21 septembre 2021. URL : http:// journals.openedition.org/ccrh/3497 ; DOI : https://doi.org/10.4000/ccrh.3497

Ce document a été généré automatiquement le 21 septembre 2021.

Article L.111-1 du Code de la propriété intellectuelle. 


\title{
Lire et écrire
}

\author{
Une enquête, un moment historiographique \\ Dominique Julia et Jacques Revel
}

1 Il n'est pas aisé, à trente ans de distance, d'apprécier ce qu'a été la nouveauté d'un livre qui est devenu très rapidement un « classique » de l'historiographie culturelle, traduit en plusieurs langues et qui a été largement intégré au bagage commun reçu dans la discipline. Pourtant, il faut bien tâcher de retrouver les conditions de sa genèse : elles se situent au point de jonction entre le renouvellement d'une historiographie tant dans ses méthodes que dans ses objets, et une conjoncture historique précise.

2 Auparavant, il peut être utile de rappeler d'un mot ce qui était sans doute le plus évident à l'époque, qui est aussi ce qui est souvent devenu étranger à nos manières de faire : au terme de trois décennies des transformations rapides qui ont bouleversé le visage de la France après la Seconde Guerre mondiale, l'exigence était fortement ressentie d'une mesure du changement, y compris au passé. C'était le temps des grandes entreprises quantitatives lancées par de grandes institutions, l'INSEE, L'INED, et qui ont aussi dominé un moment de la vie scientifique collective du Centre de recherches historiques. Pendant une trentaine d'années, ces enquêtes ont été perçues, à tort ou à raison, comme la marque de fabrique de la VI e Section de l'École pratique des hautes études, plus largement comme celle du mouvement des Annales. Ce n'est pas ici le lieu de discuter la validité de ce diagnostic sur le fond, d'autant que, est-il besoin de le rappeler, le modèle de l'enquête quantitative ou sérielle se retrouvait au même moment dans la plupart des historiographies occidentales. Retenons-en au moins la puissance et l'attrait d'une démarche dont François Furet avait fortement rappelé, peu auparavant, les avantages méthodologiques et heuristiques ${ }^{1}$.

3 À ces projets correspondait aussi une organisation collective du travail de recherche qui avait été empiriquement mise en place dans les années cinquante puis progressivement développée. Cette organisation reposait sur une participation ouverte, rassemblant des chercheuses et des chercheurs appartenant au Centre de recherches historiques (Muriel Jeorger, Véronique Nahoum, Marie-Laurence Netter, Yves Pasquet) et d'autres dont l'activité principale s'inscrivait dans d'autres configurations institutionnelles, MarieMadeleine Compère à l'Institut National de la Recherche Pédagogique (INRP), Paul Butel, 
Guy Mandon et Jean-Pierre Poussou à l'Université de Bordeaux, Pierre Lévêque à l'Université de Dijon, pour ne mentionner que les contributeurs aux deux volumes de Lire et Écrire. Cette organisation reposait aussi pour une part, ne le dissimulons pas, sur une hiérarchisation des tâches au sein de l'équipe de recherche. On en retrouve la marque lisible dans la publication finale et elle a pu, à l'occasion, nourrir des tensions entre les participants. Aussi bien ce projet abouti, réussi, a-t-il été l'un des derniers à se conformer au modèle "classique » de l'enquête, modèle qui s'est progressivement effacé au cours des années quatre-vingt pour être remplacé par d'autres formes de travail collectif.

Les deux initiateurs de l'enquête qui nous retient ont été l'un et l'autre les acteurs et les analystes de ce renouvellement. Dès les années 1969, le séminaire commun de François Furet et d'Emmanuel Le Roy Ladurie à la VI ${ }^{\mathrm{e}}$ Section de l'École pratique des hautes études (EPHE) faisait déjà la part belle à l'anthropologie culturelle et l'on y présentait et discutait les publications récentes. Jacques Ozouf avait fait paraître, en 1967, dans la collection "Archives", le petit livre appelé lui aussi à faire date, Nous, les maîtres d'école, où il restituait à ceux qu'il avait questionnés, la matière même de leurs réponses et leur proposait ses premières hypothèses de recherche ${ }^{2}$. Au cours de ces mêmes années, François Furet travaillait à l'enquête dédiée à la production de la librairie du royaume de France, dont un premier volume, Livre et société dans la France du XVIII siècle, parut dès 1965, le second étant publié en $1970^{3}$.

5 Si l'on considère la décennie 1965-1975, on peut reconnaître, parmi d'autres (qui ont été évoqués dans le bilan historiographique dirigé par Jacques Le Goff et Pierre Nora, Faire de l'histoire $)^{4}$ trois changements majeurs. On observe tout d'abord une avancée marquée des questions d'anthropologie historique de la culture. On peut rappeler ici Les paysans $d u$ Languedoc d'Emmanuel Le Roy Ladurie, où les chemins de pénétration de la confession huguenote étaient étudiés à partir d'un dépouillement rigoureux du nombre des signatures, et où l'auteur concluait à l'opposition entre un artisanat urbain passé au protestantisme et une population rurale demeurée papiste ${ }^{5}$. On peut citer également le petit livre de Robert Mandrou sur la Bibliothèque bleue, intitulé De la culture populaire aux XVII et XVIII siècles. Il soulignait à juste titre l'importance d'une "littérature» dite "populaire », formule éditoriale nouvelle proposée par les libraires troyens au plus grand nombre ${ }^{6}$. Dans ces mêmes années, Natalie Zemon Davis proposait une série d'analyses neuves sur les cultures du peuple, qui seront réunies en 1975 sous le titre Society and Culture in Early Modern France, livre traduit en France en $1979^{7}$; dès 1965, elle montrait la pénétration du protestantisme parmi les compagnons imprimeurs lyonnais dans les années $1540-1550^{8}$, et l'article du volume intitulé Printing and the People s'efforçait, à partir des hypothèses de Jack Goody, d'analyser les rapports complexes noués entre imprimé et culture populaire, en montrant les modi-fications profondes qu'entraîne dans la vie culturelle de certaines catégories urbaines (petits marchands, artisans, ouvriers semiqualifiés, domestiques) l'usage de l'imprimé9. On n'évoque enfin que pour mémoire l'enquête sur l'anthropologie du conscrit français, puisqu'il en a déjà été question dans cette journée. Mais il est clair que les données rassemblées par Michel Demonet, Paul Dumont et Emmanuel Le Roy Ladurie pour construire « une anthropologie de la jeunesse masculine au niveau d'une cartographie cantonale (1819-1830)», ont elles aussi irrigué le questionnaire de Lire et Écrire. En témoignent à suffisance les cartes publiées des conscrits répartis en trois catégories : ne sachant ni lire ni écrire, sachant lire et écrire et sachant lire seulement ${ }^{10}$. 
6 Un second déplacement majeur est venu par le biais de la démographie historique. En 1957, dans la revue de l'Institut national d'études démo-graphiques, Population, Michel Fleury et Pierre Valmary redonnaient une nou-velle jeunesse à l'enquête qu'avait conduite, aux débuts de la III ${ }^{\text {e }}$ République, le recteur Louis Maggiolo sur les signatures des époux dans les registres paroissiaux et les registres d'état-civil avec l'aide des instituteurs bénévoles qu'il avait su mobiliser. À partir de ces données, ils cartographiaient, pour la première fois, les résultats des coupes quinquennales opérées depuis la fin du XVII ${ }^{\mathrm{e}}$ siècle jusqu'en 1816-1820. Les auteurs formaient des vœux pour une reprise des recherches sur les signatures et signalaient qu'au demeurant :

les dépouillements systématiques de registres paroissiaux récemment entrepris pour l'étude des populations anciennes, comportent le relevé des signatures, et même celui de leur qualité ${ }^{11}$.

7 Il faut bien reconnaître que leur étude n'a pas reçu, au moment de sa publication, un écho considérable auprès des historiens, peut-être parce qu'elle était publiée dans une revue de démographie qu'une bonne partie d'entre eux ne lisait pas encore ${ }^{12}$. Dès l'année suivante pourtant, Michel Fleury et Louis Henry, dans un grand article prospectif, proposaient une vaste enquête sur la population de la France depuis le règne de Louis XIV; Se félicitant des résultats prometteurs obtenus par les toutes récentes monographies sur Beauvais et le Beauvaisis ${ }^{13}$ et sur Crulai $^{14}$, ils y exposaient le projet d'un dépouillement systématique des registres paroissiaux sur l'ensemble du territoire national à partir d'un échantillon rationnel au centième des communes françaises (telles qu'elles étaient définies au recensement de 1954), tiré au sort selon la théorie des sondages et stratifié par département: communes rurales, petites villes (une sur dix), villes moyennes (une sur cinq), dix grandes villes (qui font toutes partie de l'échantillon). Ce programme d'enquête à long terme qui, dans un premier temps, se fixait pour but principal l'étude du mouvement de la population, l'établissement de tables de mortalité pour le XVIII ${ }^{\mathrm{e}}$ siècle, la fixation de l'état de la population française au début du XIX ${ }^{\mathrm{e}}$ siècle et l'analyse de la fécondité par la méthode de reconstitution des familles, comportait, parmi les relevés prévus, celui des signatures des époux aux actes de leur mariage, prélude à une statistique, à l'échelle nationale, de l'évolution du degré d'accès à l'écrit ${ }^{15}$. Si la plupart des résultats démo-graphiques de l'enquête ont été publiés dès 1975 dans un numéro spécial de la revue Population ${ }^{16}$, ceux qui concernaient l'alphabétisation à partir des signatures ont fait l'objet d'un seul article publié dans la première livraison de la même revue en $1977^{17}$. Mais, parallèlement à l'enquête de l'INED, toute une série de thèses d'histoire sociale, qui appuyaient leur démonstration sur les massifs dépouillements de la démographie historique, apportaient, elles aussi, des éléments neufs sur la distribution de l'indicateur des signatures. Qu'il suffise de citer ici, sans nulle prétention à l'exhaustivité, les ouvrages de Marcel Lachiver sur Meulan ${ }^{18}$, de Maurice Garden sur Lyon ${ }^{19}$, d'Alain Corbin sur le Limousin ${ }^{20}$, de Jean-Claude Perrot sur Caen ${ }^{21}$. À cette série de travaux, il convient d'ajouter la thèse de Jean Quéniart, soutenue en 1975, sur l'histoire sociale de la culture dans neuf villes de l'Ouest, dont toute la première partie est consacrée à une analyse comparée des signatures aux actes de mariage ${ }^{22}$. Cette esquisse demeurerait incomplète si l'on n'y joignait deux grandes thèses de démographie historique urbaine, dont le développement a été contemporain de (ou légèrement postérieur à) la publication de Lire et Écrire, celle de Jean-Pierre Poussou (1978), consacrée à la croissance économique et à l'attraction urbaine de Bordeaux au XVIII ${ }^{\mathrm{e}}$ siècle ${ }^{23}$; Poussou a été, rappelons-le, l'un des collaborateurs actifs de l'enquête de François Furet et Jacques Ozouf et il a présenté, dans le second volume de celle-ci, une large synthèse des 
recherches qu'il avait conduites sur l'Aquitaine au XVIII ${ }^{e}$ siècle ${ }^{24}$. L'autre thèse, celle de Jean-Pierre Bardet sur Rouen aux XVII et XVIII ${ }^{\mathrm{e}}$ siècles, met au jour les différenciations spatiales - et donc sociales - de la répartition des signatures à l'intérieur de la ville ${ }^{25}$. En dix ou quinze ans, l'alphabétisation et son développement ont ainsi cessé d'être des objets secondaires pour faire partie désormais d'un horizon de recherche partagé par nombre d'historiens français et elle a tendu à occuper une place de plus en plus importante dans leurs problématiques. Entre 1974 et 1976, l'une des questions mises au concours de l'agrégation d'histoire - qui ne passe pourtant pas pour être l'un des lieux les plus révolutionnaires de la corporation - fut consacrée à « La famille, l'enfant et l'éducation en France et en Grande-Bretagne du XVI ${ }^{e}$ au XVIII ${ }^{e}$ siècle ». La traduction de livre célèbre de Peter Laslett sous le titre Le monde que nous avons perdu, en $1969^{26}$, la réédition, en 1973, de l'ouvrage de Philippe Ariès, L'Enfant et la vie familiale sous l'Ancien Régime, paré cette fois de la notoriété qu'il a acquise outre-Atlantique (alors que la première édition de 1960 n'avait obtenu qu'un succès limité), ne sont sans doute pas étrangères à l'entrée des thématiques qui y sont développées dans la formation des enseignants. Ce sont en tout cas les indices d'un renouvellement partiel du paysage historiographique.

Pour comprendre ce glissement, en quelques années, d'une analyse essentiellement socioéconomique, largement dominée par l'étude des prix, des salaires et des distributions sociales, à une histoire socioculturelle, il convient - et c'est le troisième point que nous souhaitions souligner - de revenir aux problématiques anglo-saxonnes. Celles-ci ont pris l'alphabétisation et, plus généralement, l'éducation comme indicateur et facteur du développement économique. À cet égard, la première étude de Lawrence Stone sur la révolution éducative en Angleterre, de 1560 à 1640, menée à partir des registres d'immatriculation des collèges universitaires d'Oxford et de Cambridge, avait, dès 1964, démontré l'accroissement soudain et sans précédent des entrées dans les universités, puisque leur niveau atteint à cette époque ne devait pas être retrouvé avant la fin du XIX ${ }^{e}$ siècle et le début du $\mathrm{xx}^{\mathrm{e}}$ siècle $^{27}$. Le socle de cette révolution éducative reposait tout à la fois sur le développement de l'imprimé comme vecteur de communication, sur la montée en puissance des États modernes, avec la croissance rapide de leurs bureaucraties, tant civiles qu'ecclésiastiques, et sur l'incitation particulière constituée par la controverse entre les confessions antagonistes qui requérait une formation approfondie des théologiens et des pasteurs. Mais, par-delà cette enquête, davantage centrée sur l'enseignement des universités, qui devait aboutir à un ouvrage en deux volumes plus $\operatorname{tard}^{28}$, c'est l'alphabétisation qui était au cœur des deux publications fondamentales en 1969. Tout d'abord, le livre de Carlo M. Cipolla, Literacy and Development in the West, ouvrage fondateur en ce qu'il appliquait au passé de l'Europe la théorie économique du capital humain, dans laquelle la dépense d'éducation représente un investissement: s'appuyant sur les statistiques nationales disponibles (recensements de population, sources militaires sur le recrutement des conscrits, etc.), il démontrait qu'une réserve d'alphabétisation (correspondant environ à $40 \%$ de la population totale, soit à plus de la moitié des hommes) était nécessaire pour qu'un pays fasse sa révolution industrielle. L'Angleterre était à la fois le pays le plus alphabétisé et le plus industrialisé de l'Europe dès la seconde moitié du XVIII ${ }^{e}$ siècle, l'Europe du Nord la suivait à quelques décennies de distance, mais l'Europe du Sud n'avait pas atteint ce taux avant 1900 et elle avait manqué sa première industrialisation ${ }^{29}$. Cette même année 1969, Lawrence Stone proposait, pour la première fois, une courbe chronologique de la progression de l'alphabétisation masculine en Angleterre, de 1640 à 1900, ainsi que les distributions géographiques et 
sociales dans la répartition des signatures, analysant les facteurs qui accélèrent ou retardent l'entrée dans l'écrit: il s'appuyait aussi bien sur les taux de signatures masculines au serment de loyauté au Parlement (Protestation oath) de 1642 que sur les trends d'alphabétisation établis soit à partir des registres de mariages, soit plutôt à partir des licences de mariage délivrées par les autorités diocésaines (dont il ne méconnaissait pas les biais sociaux qu'elles comportaient), et les travaux menés par le groupe de recherche de Cambridge sur l'histoire de la population en Grande-Bretagne, sous la direction de Roger $S$. Schofield, lui avait été particulièrement précieux ${ }^{30}$. L'année précédente en effet, ce dernier venait de publier, dans le livre collectif dirigé par Jack Goody, Literacy in Traditional Societies, l'un des tout premiers articles sur la mesure de l'alphabétisation dans l'Angleterre préindustrielle ${ }^{31}$. Tout un champ de recherche historique se voyait ainsi ouvert et c'est bien dans ce contexte intellectuel qu'il faut resituer la genèse et le développement de l'enquête qui précède la rédaction de Lire et Écrire.

D'autres sources historiographiques de cette enquête pourraient naturellement être évoquées. Il convient pourtant d'en venir à la conjoncture précise au sein de laquelle elle s'est élaborée. Il est à peine besoin de rappeler que les années soixante et le début des années soixante-dix - de part et d'autres de 1968 - ont été un moment d'intense réflexion critique sur l'institution scolaire et sur son rôle social. Les premiers travaux de Pierre Bourdieu et de Jean-Claude Passeron, le très large débat contradictoire qu'ils ont suscité et nourri ne sont sans doute ici que l'aspect le plus visible d'un mouvement de fond. Une ancienne confiance s'est alors trouvée ébranlée qui liait inséparablement l'école et la République. Ces mêmes années sont précisément celles où le regard sur l'école de la III ${ }^{e}$ République, sur ses ambitions et ses réalités, s'est fait mélancolique, parce que l'interrogation sur les pouvoirs réels dont disposent l'école et ses maîtres se fait plus vive. Temps des illusions perdues? Peut-être, et d'autant plus intensément ressenti que l'ébranlement qui a frappé l'édifice républicain et les certitudes sur lesquelles il reposait est profond. En tous les cas, les réponses qu'avaient faites les instituteurs au questionnaire que leur avait adressé Jacques Ozouf soulignaient, avec une stupeur voilée de tristesse, l'écart qui séparait à leurs yeux hier et aujourd'hui ${ }^{32}$. Un article de Jacques Ozouf lui-même éclaire bien la tonalité du désenchantement à l'œuvre au cours de la décennie soixante : il s'agit du compte rendu, rédigé à l'automne 1968, donc après les " événements", de l'ouvrage d'Antoine Prost, L'enseignement en France 1800-1967, dont l'achevé d'imprimer date de juillet $1968^{33}$. La nouveauté du livre de Prost consistait, entre autres apports, à démontrer que le conflit idéologique autour de la liberté de l'enseignement dans la première moitié $\mathrm{du} \mathrm{xIX}^{\mathrm{e}}$ siècle, autour de la laïcité dans la seconde moitié de celui-ci, masquait en réalité un accord profond des acteurs sur la fonction et les finalités de l'école. Voici ce que notait à ce propos Jacques Ozouf :

Antoine Prost a pris le parti de gommer les accidents qui, de 1800 à nos jours, hérissent l'histoire de l'enseignement, comme si les lois Ferry contredisaient moins la loi Falloux qu'elles ne la prolongent sourdement. Tout son livre sautant à pieds joints sur les conflits politiques s'attache à montrer, entre républicains et conservateurs, un accord tacite que masque l'écume provisoire des dissensions secondaires.

Même s'il prenait ses distances avec une thèse où il croit déceler,

l'espérance tenace d'une certaine gauche française qui voyait partout des catholiques en rupture avec la hiérarchie et leur accorde une importance sans cesse démentie par les faits. 
11 Jacques Ozouf accordait à l'auteur le «fond d'unanimité » sur lequel se déroulaient les querelles scolaires, la croyance partagée par les conservateurs et les libéraux qui «attendent tous de l'école qu'elle engendre une société à son image», et, non sans humour, demeurait dubitatif sur son affirmation selon laquelle cet « âge d'utopie » était désormais «déclaré révolu»: s’il est vrai que "c'est une illusion typiquement universitaire que d'attendre, d'un enseignement neuf, une société neuve », il reste que «ce rêve a la vie dure : chaque jour de cet automne le vérifie un peu plus ». Toutefois, il concluait son article par un accord sur le diagnostic porté par l'auteur :

Le livre d'Antoine Prost met à nu les racines du mal de l'Université : face à une demande sociale qui a changé, c'est la crise d'une culture demeurée immobile. Certes il y a mille remèdes, suggérés au fil de ces pages vigoureuses. Mais, de thérapeutique d'ensemble, point. L'auteur estime devoir confesser ici une incapacité radicale. Car la jeunesse, qui résume le système des techniques et ces valeurs que les adultes lui transmettent en échange de son intégration sociale, se soucie comme d'une guigne de projeter devant elle sa vie adulte. Et les adultes, d'un autre côté, ont perdu toute foi dans leurs remèdes éducatifs. Nul ne sait donc plus ce qu'il faut faire. C'est la rançon du dépérissement des idéologies ${ }^{34}$

12 Cet article a le mérite de bien mettre en lumière la conjoncture historique dans laquelle s'est élaborée l'enquête, puis a été rédigé Lire et Écrire. L'événement invite à relire autrement l'histoire de l'éducation. Le séminaire de Furet - ce n'est sans doute pas un hasard - avait été consacré en 1970-1971 à l'histoire de l'enseignement secondaire, puis, fusionnant avec celui de Jacques Ozouf, il s'était délibérément orienté, dès l'année suivante, vers une histoire de l'enseignement primaire et de l'alphabétisation dans la longue durée ${ }^{35}$. Le pouvoir politique semblait lui-même acquiescer à cette nécessité d'une relecture critique de l'histoire de l'éducation, puisqu'un haut fonctionnaire à l'histoire de l'éducation avait été nommé en juillet 1970 par Olivier Guichard, alors ministre de l'Éducation, et qu'une "Commission permanente d'histoire de l'éducation ", consacrée officiellement en juillet 1972, siégea de 1971 à 1974 pour soutenir les travaux scientifiques dans ce domaine, commission qui, après quelques péripéties, devait laisser la place à un Service d'histoire de l'éducation, rattaché à l'Institut national de recherche pédagogique, encore bien vivant ${ }^{36}$. Dans l'avertissement de Lire et Écrire, Furet et Ozouf signalent d'ailleurs avoir obtenu de la part de cette Commission un financement pour leur enquête et rendent hommage à la mémoire du recteur Maurice Bayen qui la dirigea ${ }^{37}$.

Ils soulignent d'emblée la place particulière qu'a occupée, dans la réflexion d'ensemble sur le système éducatif, l'histoire de l'alphabétisation saisi : il y a là ,

un de ces sujets brûlants qui ont mobilisé en France des passions très fortes. Car elle n'a pas été faite pour elle-même, mais a servi à argumenter le débat politique sur l'école, resté jusqu'à une date récente une des grandes lignes de clivage entre la droite et la gauche. Par là même, elle a été liée à des enjeux et à des valeurs essentiels à notre vie politique.

On aura reconnu les premières lignes de l'introduction de Lire et Écrire ${ }^{38}$. Elles font comprendre ce qu'a été l'enjeu profond de l'enquête aux yeux de ceux qui en ont pris l'initiative et la responsabilité. Pour reprendre un terme souvent utilisé à propose de l'historiographie de la Révolution française, il s'agissait de "refroidir » les enjeux idéologiques passés et présents de l'histoire de l'alphabétisation en en livrant une description et une analyse critiques :

Décrire, c'est-à-dire mesurer, établir de façon précise les rythmes du processus, sa diffusion par sexes, et par types de milieux géographiques et humains. Analyser, 
c'est-à-dire comprendre quels sont les variables principales qui expliquent le

phénomène et quelles peuvent en être les conséquences ${ }^{39}$. le plus souvent surinvestie par les passions politiques pour reprendre le dossier sur des données renouvelées ou, en tout cas, soumises à un nouvel examen. Notons qu'à cet égard, les motivations de Ozouf et de Furet ont pu être partiellement différentes au départ du projet qui les a mobilisés, avec leurs collaborateurs, pendant plusieurs années. À l'histoire de l'enseignement et de l'éducation républicaines, le premier avait, on l'a rappelé, choisi de consacrer l'essentiel de sa vie d'historien. Pour le second, il a pu s'agir plus généralement de prolonger sur un terrain nouveau sa réflexion sur les continuités et les ruptures entre l'Ancien Régime et la France issue de la Révolution, mais aussi sur les rapports entre l'institution et la société. comparables doit lui permettre de tester empiriquement la validité des thèses affrontées, ou encore d'en suggérer de nouvelles, sur le rôle de l'école dans les processus d'alphabétisation. Elle reste fidèle en cela à l'entreprise de Maggiolo un siècle auparavant, même si elle le fait bien évidemment avec des moyens techniques incomparablement plus puissants. Le recours aux démarches quantitatives ne pouvait certainement pas être perçu comme une innovation dans les années soixante-dix. Elle avait derrière elle une histoire déjà longue de pratiques et aussi de polémiques, parfois fort vives. Sur des objets différents, Furet comme Ozouf en avaient déjà une solide expérience. La nouveauté était plutôt du côté de l'utilisation d'un instrument technique, l'ordinateur, avec les possibilités qu'il offrait désormais aux historiens de traiter des masses considérables de données, et surtout le recours à une technique d'analyse statistique qui connaissait alors un succès spectaculaire chez les historiens, l'analyse factorielle des correspondances, qui rendait possible une « décomposition analytique de la réalité en niveaux de description différents $»^{40}$. L'expérimentation statistique se retrouvait ainsi explicitement placée à la base de toute proposition d'une interprétation. Elle fondait le refus d'accepter de prendre le phénomène de l'alphabétisation comme il était habituellement accepté par ses commentateurs, quels que fussent les désaccords qui les affrontaient: comme un phénomène global et univoque.

Sur le traitement statistique dont leurs données ont fait l'objet et en vue duquel elles ont été recueillies, les deux auteurs ne se sont pas arrêtés outre mesure dans l'ouvrage qui est issu de leur enquête ${ }^{41}$. On peut sans doute y voir le résultat d'une division du travail à un moment où des spécialistes pouvaient encore être mis à la disposition des historiens et les accompagner dans leur démarche. Il est probable aussi que Jacques Ozouf, praticien éprouvé de la statistique électorale, avait acquis en ce domaine une familiarité qui pouvait l'inciter à faire l'économie des attendus que l'on retrouve parfois lourdement présents chez les premiers historiens utilisateurs de l'analyse factorielle ${ }^{42}$. Il reste que l'ensemble du projet, et sa réalisation finale, donnent le sentiment d'une confiance de fond dans les instruments d'analyse et de mesure désormais disponibles et sur le contrôle qu'ils permettent d'exercer sur des données discontinues, souvent ambiguës et longtemps tenues pour incertaines. Rien, sans doute, ne le fait mieux voir que le titre retenu pour le chapitre 6 du premier volume : "Le rôle de l'école: Le verdict de l'ordinateur ", qui rassemble les arguments raisonnés d'une conclusion d'ensemble. On reviendra plus loin sur le détail de la démonstration. Retenons pour l'instant la confiance indiscutée que manifestait cet effet d'annonce, et la conviction qu'elle exprimait de pouvoir fonder une 
réponse positive, factuelle sur une démarche contrôlée de bout en bout. En ce sens, Lire et Écrire n'a pas seulement été l'une des dernières grandes enquêtes quantitatives menée à bien au sein du Centre de recherches historiques. Le livre plaidait aussi pour une histoire dont les résultats seraient cumulatifs, et il le faisait avec un optimisme qui allait être fortement révisé à la baisse à la faveur des turbulences des années suivantes et qui peut nous paraitre bien lointain.

Ces rappels pouvaient n'être pas inutiles pour replacer l'enquête dans le moment historiographique qui a été le sien. Mais il est temps d'entrer maintenant dans la démonstration qu'elle propose.

Elle opère un double déplacement tout à fait fondamental par rapport à l'historiographie de l'enseignement. Le premier est le choix délibéré de la longue durée, qui vise à manifester les continuités qui perdurent par-delà la coupure révolutionnaire. Ce renversement d'optique constitue un choix particulièrement judicieux dans ce secteur extrêmement sensible de l'historiographie. Il fait en effet litière de tout l'héritage qui avait vu s'opposer, surtout entre 1870 et 1914, historiens cléricaux et historiens républicains, les premiers s'attachant à manifester l'antériorité de l'œuvre pédagogique de l'Église après le concile de Trente, tandis que les seconds préféraient voir dans les décrets des assemblées de la Révolution les lointains ancêtres des lois Ferry, après plus d'un siècle de combats acharnés pour arracher l'enfance à l'emprise des curés, des sœurs et des frères enseignants. Or, si la Révolution a bien fait de l'école un lieu «central d'affrontement politique et culturel », et si elle est bien à l'origine de notre idéologie contemporaine de l'école, elle n'a pu, en revanche, mettre en œuvre une réelle politique de l'école élémentaire. Les coups de sonde opérés par le livre, à partir des enquêtes révolutionnaires de l'an VI et de l'an VII dans onze départements, confirment plutôt l'explosion de l'initiative privée sous la Révolution ${ }^{43}$ : l'École normale de l'an III n'a d'ailleurs pas été un vivier d'instituteurs d'écoles élémentaires comme le prévoyait son décret de fondation qui fixait comme mission aux élèves, une fois rentrés dans leurs districts, d'ouvrir des écoles normales "secondes", où ils formeraient les futurs instituteurs, mais bien plutôt un foyer de recrutement des futurs professeurs d'écoles centrales ${ }^{44}$. L'ouvrage de Furet et Ozouf gomme donc délibérément ce qui avait constitué le credo de toute une historiographie républicaine. Bien plus, il regarde les lois de Jules Ferry comme un point d'arrivée et non comme un point-origine ou avènement. Il y a là un renversement décisif de perspective qui refuse de céder à «l'illusion du politique » ${ }^{45}$, et qui bouleverse ainsi le récit canonique que véhiculait une écriture traditionnelle de l'histoire de l'éducation.

En mettant au cœur de leur dispositif d'étude l'analyse de la croissance de l'alphabétisation comme indicateur d'un processus d'expansion de plus en plus large de la culture écrite, et en restituant à la demande sociale d'éducation un rôle majeur, les auteurs opèrent un second déplacement, tout aussi majeur que le premier. Non que l'école soit absente de l'ouvrage puisque trois chapitres - soit plus de $40 \%$ du livre - lui sont consacrés ${ }^{46}$. Mais ils s'interrogent d'abord sur les inégalités comme sur les voies d'accès à la culture écrite, sur les discontinuités du rythme de l'alphabétisation faites de progressions mais aussi de paliers et de reculs, sur les dénivellations sociales et régionales qu'il est possible de repérer. C'est, contre toute une tradition historiographique antécédente, mettre en question l'évidence de l'école, sa nécessité, son rôle décisif dans la constitution de ces différences et de ces variations vis-à-vis de la culture écrite; et les auteurs tirent sans doute des conclusions excessives des calculs des coefficients de 
corrélation qu'ils ont établis entre alphabétisation et scolarisation ${ }^{47}$. Mais leur mérite essentiel reste d'avoir su restituer une dynamique sociale dans toute sa complexité plutôt que d'imaginer une efficacité mécanique de l'école qui agirait par elle-même dès lors que celle-ci a été fondée. C'est ici que l'hypothèse d'une circularité entre alphabétisation et scolarisation ${ }^{48}$ manifeste toute sa fécondité : c'est la demande sociale d'instruction qui pousse à la création des écoles, à leur fréquentation progressivement plus régulière, et qui produit, avec la scolarisation, l'alphabétisation. À mesure que celle-ci s'étend, elle apparaît de plus en plus nécessaire et il devient déshonorant de ne pas maîtriser la lecture et l'écriture. D'où la demande de fondation de nouvelles écoles dont la présence fait à nouveau progresser l'alphabétisation.

21 C'est ce double renversement de perspective qui donne cohérence et puissance à l'ouvrage. À quoi il convient d'ajouter le jeu continuel qui, mettant en œuvre des échelles d'analyse différentes, autorise la compréhension des dynamiques à l'œuvre, depuis un niveau macroscopique jusqu'à l'échelon local : il y a, d'une part, les analyses factorielles menées sur les statistiques de signatures de l'enquête de Louis Maggiolo, les distributions géographiques des informations recueillies dans les recensements de 1866 et 1872 (qui distribuaient la population en trois groupes par rapport à l'instruction : ne sachant ni lire ni écrire, sachant lire seulement, sachant lire et écrire, le recensement de 1872, qui permettait en outre une grossière répartition par groupes d'âge) et des renseignements contenus dans les statistiques de recrutement des conscrits (qui fournissaient les mêmes données depuis 1827$)^{49}$; il y a, d'autre part, les apports des dépouillements opérés dans les archives départementales et communales, dont les résultats sont rassemblés dans les contributions du second volume et qui servent également à la démonstration synthétique du premier ${ }^{50}$. Par le retour aux sources locales, l'enquête collective autorise un changement de focale et permet d'examiner à la loupe des fonctionnements qui, autrement, seraient demeurés méconnus. Au reste, l'enquête à laquelle avaient participé, en 1877-1880, quelque 16000 instituteurs bénévoles réquisitionnés par le recteur Louis Maggiolo, souffrait de trois biais dans les relevés effectués, qui venaient en obérer les résultats. Tout d'abord, un certain nombre de départements n'avaient pas été pris en compte pour les coupes quinquennales de signatures qui devaient couvrir l'ensemble du territoire: au premier chef, les départements d'Alsace-Lorraine annexés au Reich allemand après la défaite de 1871 (Bas-Rhin, Haut-Rhin, parties des départements de la Meurthe, de la Moselle et même des Vosges), mais aussi des départements plus ou moins récemment rattachés au territoire français comme la Corse, le Vaucluse (l'ancien Comtat Venaissin papal), la Savoie et la Haute-Savoie, et surtout la Seine, dont les registres de baptêmes, mariages et sépultures, mais aussi les registres d'état-civil avaient brûlé lors de l'incendie de l'Hôtel de Ville, le 24 mai 1871. En second lieu, la base statistique de certains relevés départementaux restait insuffisante, voire dérisoire ${ }^{51}$. Enfin, les scores des populations urbaines avaient été systématiquement sous-évalués: il est vrai que les relevés étaient beaucoup plus longs à effectuer dans les grandes villes, mais, du même coup, les villes étant, en règle générale, en avance sur les campagnes environnantes (même s'il existe des exceptions), les scores globaux d'alphabétisation se trouvaient du même coup sous-évalués. Les contributions régionales ou locales du second volume ont donc constitué le laboratoire où pouvaient être testées et vérifiées les hypothèses qui ont nourri la démonstration synthétique du premier volume. C'est ce va-et-vient constant d'une analyse à l'échelle du territoire national tout entier, et l'étude de terrains locaux bien délimités qui donne cohérence et solidité au dispositif, en prenant le pari de la complexité, ce qui est un plaidoyer pour l'intelligence des phénomènes ${ }^{52}$. 
Soient ici quelques exemples : les contrastes d'alphabétisation que Pierre Lévêque repère en Bourgogne entre l'openfield de la Côte-d'Or et le bocage des arrondissements d'Autun et de Charolles ${ }^{53}$ viennent nourrir le chapitre 4 du tome I consacré au paysan entre l'oral et l'écrit, tout comme un mémoire de maîtrise soutenu en 1973 à l'Université de Tours par Jean-Paul Giret, qui a analysé dans le département de l'Eure-et-Loir l'opposition du degré de l'alphabétisation des conscrits issus de la Beauce par rapport à celui des conscrits nés dans le Perche ${ }^{54}$. Mais, en réalité, il serait trop simple d'identifier le bocage à l'analphabétisme et l'openfield au progrès de l'instruction, et les auteurs savaient trop bien qu'ils risquaient de s'exposer au reproche naguère adressé à André Siegfried pour avoir véhiculé dans le Tableau politique de la France de l'Ouest le dicton populaire selon lequel «le granit produit le curé et le calcaire l'instituteur "55. L'analyse réalisée par Muriel Jeorger sur le diocèse de Rouen montre qu'il existe des bocages bien alphabétisés : elle décortique les raisons qui pouvaient rendre compte de l'avance du pays de Bray, pays de bocage surpassant tous les autres secteurs régionaux: l'absence d'industrie textile, l'existence de communaux étendus, une propriété rurale assez bien distribuée et une classe de paysans propriétaires ou fermiers vivant d'une production commercialisable ont été autant de facteurs qui peuvent rendre compte d'une dynamique qui s'est poursuivie pendant le $\mathrm{XIX}^{\mathrm{e}}$ siècle. Parallèlement à l'étude différentielle des signatures, l'analyse des procès-verbaux des visites pastorales, tant dans le diocèse de Rouen que dans celui de Montpellier au XVIII siècle, met au jour les relations complexes qui se nouent entre les dynamiques de l'implantation scolaire et celles de l'alphabétisation ${ }^{56}$ : la présence ou la fondation d'écoles n'est pas signe de leur efficacité, même si l'on peut retrouver ici ou là des traces tangibles d'une politique scolaire épiscopale ${ }^{57}$, ni d'une fréquentation différenciée socialement (en Languedoc, l'école semble avoir davantage été utilisée par les fils des notables), ni non plus d'une assiduité régulière, l'absentéisme étant l'un des fléaux les plus courants. Quant à l'avance dans le processus d'entrée dans la culture écrite, que pourrait constituer l'imprégnation protestante, elle paraît bien atténuée au XVIII ${ }^{\mathrm{e}}$ siècle puisqu'à niveau social équivalent, les scores d'alphabétisation des catholiques et des protestants sont voisins : il vaut mieux souligner que l'émulation entre communautés de confession différente a largement compté pour permettre, au XVIII ${ }^{e}$ siècle, un développement scolaire au sein du «triangle fatal » d'ignorance que décrivait un peu plus tard Stendhal ${ }^{58}$. Les analyses microscopiques, par la complexité des situations locales qu'elles révèlent, interdisent donc toute interprétation univoque.

L'enquête de Louis Maggiolo avait délibérément laissé en retrait l'étude de l'alphabétisation dans les villes, et privilégié les campagnes. La relecture d'une bibliographie parfois ancienne, la prise en compte des données récemment recueillies pour les thèses d'histoire sociale urbaine et la contribution de Jean-Pierre Poussou sur l'évolution de l'alphabétisation dans soixante-douze villes d'Aquitaine, ont offert à Furet et Ozouf la possibilité d'hypothèses fortes. Par exemple, la reprise attentive du livre du comte Eugène Fontaine de Resbecq, sur l'histoire de l'instruction primaire dans le département du Nord, qui datait de presque un siècle $(1878)^{59}$, mise en rapport avec les données statistiques du XIX ${ }^{\mathrm{e}}$ siècle (instruction des conscrits en 1827-1829, 1878-1880, 1899-1904, et signatures au mariage en 1866), permet de mesurer l'impact de la révolution industrielle sur le processus d'alphabétisation, les retards que celle-ci entraine par la mise au travail précoce des enfants ${ }^{60}$, et rejoint les conclusions auxquelles parvenait, au même moment, Michael Sanderson pour l'évolution de l'éducation élémentaire dans le Lancashire entre 1780 et $1840^{61}$. Il conviendrait d'ailleurs d'examiner plus avant une 
hypothèse émise sur le mode interrogatif par Poussou (et non reprise dans le tome I) : constatant une poussée des scores d'alphabétisation masculins sous la Révolution en Aquitaine, il se demande si celle-ci, tant dans les villes que dans les bourgs et les paroisses rurales, ne pourrait être liée au "rôle considérable des clubs et des sociétés de pensée »" ${ }^{62}$. La suggestion mériterait à tout le moins d'être testée à partir de l'inventaire des sociétés politiques dressé par Jean Boutier et Philippe Boutry ${ }^{63}$.

Les résultats de l'enquête, dont Lire et Écrire est l'aboutissement, ont été, on l'a dit, très rapidement intégrés au patrimoine commun des historiens. On voudrait, pour conclure cette brève présentation, en retenir seulement trois traits essentiels. Tout d'abord, Lire et Écrire est un livre consacré aux discontinuités temporelles des rythmes de l'évolution de l'alphabétisation dans la longue durée et aux inégalités spatiales entre deux France culturelles, et à l'intérieur de chacune de ces deux France, entre régions, entre villes et campagnes (avec le rattrapage, dès le XVIII siècle, mais surtout dans la première moitié $\mathrm{du} \mathrm{XIX}^{\mathrm{e}}$ siècle, du Midi. C'est aussi un livre attentif aux inégalités entre hommes et femmes, garçons et filles, et surtout aux inégalités sociales. Car c'est, en définitive, la stratification sociale qui est le facteur explicatif le plus puissant pour rendre compte des inégalités d'accès à la culture écrite : c'est elle qui permet de comprendre l'avance globale et générale de la ville ancienne parce que s'y concentrent les richesses et les pouvoirs, parce qu'y sont plus nombreuses les catégories sociales qui vivent de la culture écrite, parce qu'y sont plus développés aussi les moyens d'instruction, d'où sa domination; c'est elle aussi qui éclaire le retard - souvent observé mais, on l'a vu, non universel - du bocage, parce que la condition misérable du métayer y est plus fréquente, et le récit d'Émile Guillaumin, La vie d'un simple, publié en 1904, donne, avec le personnage de Tiennon Bertin, corps et chair à cette figure dominée que les propriétaires veulent maintenir dans l'ignorance: les scores abstraits d'alphabétisation prennent ici un sens précis et concret ${ }^{64}$. $\mathrm{Si}$, dans cette histoire, la religion, et surtout la division de l'ancienne chrétienté en confessions plurielles, ont joué un rôle non négligeable - l'antériorité de l'introduction de l'écriture dans les communautés réformées n'est pas contestée, pas plus que l'ampleur de la politique scolaire des évêques qui ont mis en œuvre, surtout entre 1670 et 1730, la réforme post-tridentine - ce rôle n'est pas premier : plus qu'une avance systématique des protestants sur les catholiques, dont le postulat sort ébranlé, c'est la proximité territoriale et la concurrence entre communautés de confession différente qui ont pu exercer une efficacité à cet égard.

En second lieu, Lire et Écrire, on l'a déjà évoqué, remet en question l'idée reçue d'un rapport causal et univoque qui irait de la scolarisation à l'alphabétisation. Des contreexemples et des discordances fortes ont été en effet repérés, tel le cas de l'actuel département des Basses-Alpes (Vallouise, vallée de Barcelonnette, Briançonnais) où des taux très élevés d'alphabétisation masculine se conjuguent avec l'absence d'écoles institutionnalisées ${ }^{65}$. Le savoir élémentaire se transmet ici par des circuits privés: les maîtres se déplacent de hameau en hameau, parfois pour des prestations courtes, et les habitants sont allés louer leurs services à la foire de la Saint-Martin de Gap ; parfois aussi, la transmission a pu être purement familiale, comme le rapporte le colporteur JeanJoseph Esmieu, né en 1762 à Gaudissard, hameau de Méolans dans la vallée de Barcelonnette : « Mon père m'avait appris à connaître les lettres et je commençais à faire sonner quelques syllabes, mais je ne pouvais pas encore apprécier ce que c'était d'être instruit ", écrit-il lorsqu'il évoque son départ de la maison paternelle en décembre $1773^{66}$. Ces modes individuels d'apprentissage domestique n'ont certainement pas été 
majoritaires dans la France d'Ancien Régime, et il est difficile de parvenir à bien documenter leur fonctionnement : mais entre père et fils, servante et fils du maitre, compagnons et apprentis à l'atelier, voire entre bergers - comme le raconte Valentin Jamerey-Duval à propos de son expérience en Lorraine - une relation familiale ou un compagnonnage professionnel ont pu déclencher le processus de déchiffrage tant à la ville qu'en campagne ${ }^{67}$. À l'inverse, la formation d'une bonne implantation scolaire peut coïncider avec un retard relatif de l'alphabétisation qui dure jusqu'à la fin du siècle : tel est le cas de l'Aquitaine, des pays vendéens et des départements qui sont aux frontières de la France alphabétisée comme la Sarthe, la Mayenne ou le Loir-et-Cher. Ce qui compte en définitive est la demande sociale d'instruction et le rôle des communautés d'habitants qui ont été les soutiens - ou non - de l'école ancienne dans son financement et sa continuité $^{68}$. Le renouveau actuel de l'histoire rurale, qui s'attache désormais à analyser de manière plus fine les fonctionnements différenciés de ces institutions politiques de base (communautés municipales et communautés paroissiales par exemple) devrait permettre aujourd'hui de reprendre une étude plus approfondie de la fonction stimulante ou retardatrice qu'elles ont exercée à l'égard de l'implantation scolaire : car, si la police de l'école était bien du ressort de l'évêque, son financement appartenait aux habitants ${ }^{69}$. D'où l'importance de la richesse foncière des villages, du volume de leur population respective, de la structure de l'habitat. Les communautés se sont appropriées - ou non - à leur profit un modèle proposé par les Églises et lui ont assigné un but qui n'est plus seulement celui de l'accès du chrétien aux Écritures et aux dogmes par le biais du catéchisme.

Enfin, les historiens modernistes que nous sommes peuvent bien évidemment s'interroger sur le rapport entre la fécondité des hypothèses proposées dans Lire et Écrire et les questions qui demeurent autour de la validité du test de la signature : s'il est vrai qu'en 1866 et 1872 celui-ci mesure bien une capacité à lire et à écrire (comme l'indiquent les analyses factorielles et les coefficients de corrélation établis à partir des données des recensements, des signatures aux actes de mariage, et des statistiques recueillies sur l'instruction des conscrits), que représente-t-il exactement pour les périodes où nous savons que les apprentissages de la lecture et de l'écriture n'étaient pas simultanés mais successifs? Il rassemble très certainement une population qui sait lire - ou tout au moins déchiffrer un texte, mais dont il n'est pas sûr qu'elle sache dans son ensemble écrire, soit qu'elle ait perdu très tôt l'usage de cette compétence, soit que la signature ait été le stade ultime de son apprentissage et s'apparente davantage à un dessin qu'à une écriture ${ }^{70}$. Quoi qu'il en soit de cette interrogation à laquelle aucune réponse définitive ne peut être apportée, l'identification par les auteurs d'un apprentissage spécifique du lire seulement, à base de fidélité religieuse est sans doute l'une des découvertes les plus fortes du livre: l'étude des données des recensements de 1866 et 1872 ne permet aux auteurs de saisir son extension que dans un aspect déjà résiduel, comme s'il s'agissait de buttes témoins d'un passé révolu. Mais ils en soulignent, à juste titre, deux caractéristiques fondamentales : d'une part la différenciation sexuelle qu'il enregistre puisqu'il touche davantage les filles que les garçons; d'autre part, sa géographie puisque les départements où le pourcentage de femmes sachant lire seulement dépasse $20 \%$ sont ceux du rebord Est/Sud-Est du Massif Central, les Hautes et les Basses Pyrénées, quatre départements alpins et trois départements de l'Ouest bocager (Ille-et-Vilaine, Côtes-du-Nord, Mayenne). Ils y voient avec raison la survivance d'un type d'acculturation progressivement recouvert au long du $\mathrm{XIX}^{\mathrm{e}}$ siècle par une formation scolaire plus complète, acculturation conduite par des pieuses filles comme les Béates dans le Velay, ou les Filles de l'Union ou les Filles du 
Travail dans le diocèse de Rodez. Ils montrent d'ailleurs la concordance saisissante de cette géographie avec la carte de la pratique religieuse en France, telle que l'avait établie l'abbé Fernand Boulard en $1947^{71}$. Comparaison n'est pas raison, pourrait-on objecter. Pourtant, la coïncidence des deux cartographies fait singulièrement réfléchir sur les rapports extrêmement diversifiés et complexes entretenus avec l'imprimé jusque tard dans le XIX ${ }^{\mathrm{e}}$ siècle. Les études menées sur l'implantation de l'école républicaine montrent bien l'incompréhension réciproque de deux cultures désormais antinomiques. Quand en 1880, l'inspecteur Pierre Leysenne est envoyé par Jules Ferry en Haute-Loire pour faire un rapport sur les Béates, il met bien en valeur tout ce qui sépare ces pieuses filles du modèle de l'institutrice qu'il s'agit de mettre en place :

On a beaucoup dit que la béate est une institutrice. Sans doute est-elle institutrice à certaines heures et à sa façon, mais elle est bien autre chose encore. Elle enseigne la dentelle aux jeunes filles et le catéchisme aux enfants des deux sexes. Auxiliaire avouée du curé, elle préside aux prières communes et à tous les exercices de la piété pour lesquels un prêtre n'est pas indispensable, comme le chemin de croix, le chant des vêpres, la lecture de la messe, les offices de l'Avent, du Carême et du mois de Mai, etc. Elle garde les malades, fait de la médecine et de la pharmacie; enfin veille et ensevelit les morts [...] Faut-il s'étonner que ces modestes filles [...] soient plus préoccupées des services qu'elles ont à rendre à l'Église que de l'enseignement de la grammaire et du calcul? Ne leur faisons donc aucun crime, ni d'être là, ni d'enseigner si peu. Elles n'ont que le malheur de ne plus répondre aux besoins des temps nouveaux et d'être un obstacle inconscient à un développement plus rapide de l'instruction primaire dans la Haute-Loire [...] Quant à la lecture, on peut hardiment affirmer que la plupart des Béates ne la savent pas, du moins comme on l'entend aujourd'hui dans nos écoles, et comme on devrait toujours l'entendre. Elles se bornent à faire assembler des lettres et réunir des sons, mais les enfants qui sortent de leurs écoles ne peuvent lire bien couramment que dans le catéchisme qu'ils savent par cœur et dans l'Évangile qu'ils ont lu cent fois. Il y a peu de Béates qui se hasardent jusqu'à l'écriture ${ }^{72}$.

27 Dans le portrait ainsi dressé, on perçoit bien tout l'écart qui sépare - et le choc qui en découle - la culture d'une communauté rurale à base d'oralité, de piété et de travail de la dentelle, de la mission d'instruction publique d'un inspecteur parisien. On saisit aussi combien l'apprentissage de la seule lecture dans un éventail très restreint de livres mémorisés ne permet pas d'inférer un spectre large de compétences chez les élèves. Le but de la lecture dans le système ancien est moins un accès à la compréhension du texte qu'une oralisation sans erreur, par déchiffrage ${ }^{73}$.

Il n'était pas dans notre propos d'examiner ici la réception de Lire et Écrire. Il est clair que sa publication, sa traduction rapide en langue anglaise ${ }^{74}$ ont dominé le champ des études sur l'alphabétisation tant en Europe qu'aux États-Unis. Non seulement de très nombreuses rencontres se sont tenues sur ce thème, mais toute une série de livres ont été publiés au cours de la décennie quatre-vingt/quatre-vingt-dix, qui proposaient des pistes de travail et des premiers bilans sur des pays qui étaient jusqu'alors demeurés en retrait dans ce domaine d'étude comme l'Allemagne, l'Italie ou l'Espagne ${ }^{75}$. Lire et Écrire a donc, par sa large diffusion, joué le rôle d'un vigoureux stimulant pour la recherche internationale sur l'alphabétisation. Au-delà, ce à quoi invitait surtout l'ouvrage, et notamment sa très dense conclusion «Trois siècles de métissage culturel $»^{76}$, c'est à comprendre comment l'accession à la culture écrite avait été à l'origine d'une véritable mutation anthropologique. Car c'est l'écrit qui permet de distinguer entre histoire et mémoire, qui désagrège le rapport de l'individu à la tradition orale de sa communauté d'origine pour le rattacher à un groupe plus vaste mais moins enraciné. Le livre invitait 
donc à réfléchir non seulement sur les pratiques de lecture - du simple déchiffrement à la lecture aisée, de la lecture à haute voix au sein d'un groupe à la lecture silencieuse et individuelle dans le calme du cabinet - mais aussi à examiner les variations de la relation à l'écriture et des productions qui accompagnent sa maitrise, depuis les remarques marginales inscrites sur les livres aux recueils recopiés de loci communes, aux chroniques et aux journaux personnels, tous ces ego-documents auxquels les historiens portent un intérêt de plus en plus marquép ${ }^{77}$. Il ne s'agit pas seulement de découvrir comment l'individu moderne s'est constitué et comment la pratique de l'écrit a modifié ses manières de penser; il nous faut aussi mieux connaitre la fréquence ou l'absence de recours à l'écrit dans les suppliques et les pétitions que les communautés adressent à leur seigneur ou à l'autorité administrative de l'État ${ }^{78}$. Ici encore, on le voit, Lire et Écrire a indiqué des pistes qui restent aujourd'hui ouvertes aux chercheurs.

\section{NOTES}

1. François Furet, "L'histoire quantitative et la construction du fait historique ", Annales ESC, XXVI, 1, 1971, p. 63-75.

2. Jacques Ozouf, Nous, les maîtres d'école. Autobiographies d'instituteurs de la Belle Époque, Paris, Gallimard-Julliard, coll. « Archives », 1967.

3. Geneviève Bollème, Jean Ehrard, François Furet, Denis Roche, Jacques Roger, Livre et société dans la France du XVIII siècle, Paris-La Haye, Mouton, 1965. Voir particulièrement, F. Furet, «La "librairie" du royaume de France au XVIII ${ }^{\mathrm{e}}$ siècle ", p. 3-32, in Marie-Thérèse Bouyssy, Julien Brancolini, Jean-Louis Flandrin, Maria Flandrin, Alessandro Fontana, François Furet, Denis Roche, Livre et société dans la France du XVIII ${ }^{e}$ siècle, t. 2, Paris-La Haye, Mouton, 1970.

4. Faire de l'histoire, Jacques le Goff, Pierre Nora (dirs.), Paris, Gallimard (coll. « Bibliothèque des histoires »), 3 vol., 1974.

5. Emmanuel Le Roy Ladurie, Les paysans de Languedoc, Paris, SEVPEN, 1966, p. 341-348.

6. Robert Mandrou, De la culture populaire aux XVII et XVIII siècles. La Bibliothèque bleue de Troyes, Paris, Stock, 1964.

7. Natalie Zemon Davis, Society and Culture in Early Modern France: Eight Essays, Londres, Duckworth, 1965 ; trad. fr., Les cultures du peuple. Rituels, savoirs et résistances au XVI ${ }^{e}$ siècle, Paris, Aubier-Montaigne, 1979.

8. Il s'agit du chapitre "Grève et salut à Lyon", dans la traduction française de l'ouvrage précédent, p. 15-39, paru d'abord dans Archiv für Reformationsgeschichte, LVI, 1965, p. 48-64, sous le titre « Strikes and Salvation at Lyon ».

9. Natalie Zemon Davis, Society and Culture, op. cit., p. 189-226 et 326-336 (dans la trad. fr., p. 308-365).

10. Emmanuel Le Roy Ladurie, Michel Demonet, Paul Dumont, «Anthropologie de la jeunesse masculine en France au niveau d'une cartographie cantonale (1819-1830) », Annales ESC, 31, 1976, p. 709-711. Voir déjà Jean-Paul Aron, Paul Dumont, Emmanuel Le Roy Ladurie, Anthropologie du conscrit français d'après les comptes numériques et sommaires du recrutement de l'armée (1819-1826), présentation cartographique, Paris-La Haye, Mouton, 1972, cartes 52, 53, 54, p. 174, 178, 183.

11. Michel Fleury, Pierre Valmary, «Les progrès de l'instruction élémentaire de Louis XIV à Napoléon III », Population, $12^{\mathrm{e}}$ année, 1957, p. 71-92 (citation : p. 90). Pour la période 1871-1875, les 
auteur préfèrent utiliser les données de la Statistique générale de la France aux données recueillies pour la période 1872-1876 par le recteur Maggiolo. L'enquête de Louis Maggiolo avait été publiée dans un tiré-à-part de 8 pages intitulé Ministère de l'Instruction publique. Statistique rétrospective. État récapitulatif et comparatif indiquant, par département, le nombre des conjoints qui ont signé l'acte de leur mariage au XVII ${ }^{e}$ XVIII et XIX ${ }^{e}$ siècle, documents fournis par 15928 instituteurs, recueillis et classés par M. Maggiolo, recteur honoraire, chargé d'une mission spéciale par M. le ministre de l'Instruction publique (extrait de la "Statistique de l'instruction primaire", $I^{e}$ volume), s. d. [1880].

12. L'ouvrage de Georges Duby et Robert Mandrou, Histoire de la civilisation française (Paris, Armand Colin, 1958), ne souffle mot de cette question, pas plus que l'Introduction à la France moderne 1500-1640. Essai de psychologie historique, Paris, Albin Michel, 1961 de Robert Mandrou. Il faut attendre en fait La Civilisation de l'Europe des Lumières de Pierre Chaunu (Paris, Arthaud, 1971, p. 142-151), pour qu'elle soit abordée dans un ouvrage de synthèse.

13. Pierre Goubert, Beauvais et le Beauvaisis de 1600 à 1730 Contribution à l'histoire sociale de la France du XVII ${ }^{e}$ siècle, Paris, SEVPEN, 1960. Marcel Reinhard fait un compte-rendu de cette thèse dès 1958 : « La population française au XVII e siècle », Population, 13e année, 1958, p. 619-630.

14. Étienne Gautier, Louis Henry, La population de Crulai, paroisse normande, Paris, PUF, 1958.

15. Voir Michel Fleury, Louis Henry, «Pour connaître la population de la France depuis Louis XIV. Plan de travaux par sondage », Population, $13^{\text {e }}$ année, 1958, p. 663-686. Rappelons que les mêmes auteurs avaient publié, dès 1956, Des registres paroissiaux à l'histoire de la population. Manuel de dépouillement et d'exploitation de l'état-civil ancien, Paris, INED, où ils proposent (p. 57) le relevé de la fréquence des signatures des époux aux actes de mariage et de leur qualité «à des époques assez éloignées (par exemple fin du XVII ${ }^{\mathrm{e}}$ siècle, milieu du XVIII ${ }^{\mathrm{e}}$, fin du XVIII ${ }^{\mathrm{e}}$ siècle) ». Voir aussi Jacques Houdaille, «État d'avancement d'une enquête sur la population de la France de Louis XIV à la Restauration », Population, 20e année, 1965, p. 293-295.

16. Population, $30^{\mathrm{e}}$ année, novembre 1975, numéro intitulé Démographie historique, p. 15-42.

17. Jacques Houdaille, «Les signatures au mariage de 1740 à 1829 ", Population, $32^{\mathrm{e}}$ année, 1977, p. 65-90; voir également l'article ultérieur du même auteur, "Les signatures au mariage, 1670-1739 ", Population, $43^{\mathrm{e}}$ année, 1988, p. 208-212.

18. Marcel Lachiver, La population de Meulan du XVIII au XIXe siècle (vers 1690-1870), étude de démographie historique, Paris, SEVPEN, 1969, p. 65-67.

19. Maurice Garden, Lyon et les Lyonnais au XVIII siècle, Paris, Les Belles Lettres, 1970, p. 242-243, 246-247, 254-255, 265-266, 309-313, 350-353, 450-452.

20. Alain Corbin, Archaïsme et modernité en Limousin au XIX ${ }^{e}$ siècle, 1845-1880, t. 1, «La rigidité des structures économiques, sociales et mentales », Paris, Marcel Rivière, 1975, p. 321-362 ; du même auteur, "Pour une étude sociologique de la croissance de l'alphabétisation au XIX ${ }^{\mathrm{e}}$ siècle. L'instruction des conscrits du Cher et de l'Eure-et-Loir (1833-1883) », Revue d'histoire économique et sociale, t. 53, 1975, p. 99-120.

21. Jean-Claude Perrot, Genèse d'une ville moderne. Caen au XVIII ${ }^{e}$ siècle, t. 1, Paris-La Haye, Mouton, 1975, p. 307-311.

22. Jean Quéniart, Culture et société urbaines dans la France de l'Ouest au XVIII ${ }^{e}$ siècle, Lille, Atelier de reproduction des thèses de l'université de Lille III, 1977, p.112-277. Cette thèse a paru sous le même titre, l'année suivante, à Paris, chez Klincksieck.

23. Jean-Pierre Poussou, Bordeaux au XVIII siècle. Croissance économique et attraction urbaine, Paris, Éditions de l'École des hautes études en sciences sociales, 1983.

24. Jean-Pierre Poussou, "Recherches sur l'alphabétisation de l'Aquitaine au XVIII siècle ", in François Furet, Jacques Ozouf, Lire et Écrire, op. cit., t. 2, p. 294-351.

25. Jean-Pierre Bardet, Rouen aux XVII et XVIII siècles. Les mutations d'un espace social, Paris, Société d'édition de l'enseignement supérieur, 1983, t. 1, p. 241-245, t. 2, tableaux n 103-104, p. 124-125. 
26. Peter Laslett, The World we have lost, Londres, Methuen, 1965 ; trad. fr., Paris, Flammarion, 1969.

27. Lawrence Stone, "The educational revolution in England 1560-1640 », Past and Present, 28, juillet 1964, p. 41-80. Seules les pages 41-47 concernent les écoles.

28. vol. 1, The University in society, Oxford and Cambridge from the 14th to the early 19th century; vol. 2, Europe, Scotland and the United States from the 16th to the 20th Century, Lawrence Stone (ed.), Princeton, Princeton University Press, 1974.

29. Carlo M. Cipolla, Litaracy and Development in the West, Baltimore, Penguin Books, 1969.

30. Lawrence Stone, «Literacy and education in England, 1640-1900 ", Past and Present, 42, février 1969, p. 69-139; voir aussi Roger S. Schofield, « Dimensions of Illiteracy 1750-1850», Explorations in Economic History, 10, 1973, p.437-454, où l'auteur fournit la courbe de l'alphabétisation masculine et féminine à partir d'un échantillon de 274 registres paroissiaux pour la période 1754-1840.

31. Roger S. Schofield, "The measurement of literacy in pre-industrial England ", in Literacy in Traditional Societies, J. Goody (ed.) Cambridge, Cambridge University Press, 1968, p. 311-325 ; Id., "Illiteracy in pre-industrial England : the work of Cambridge group ", Educational Reports Umea 2, 1973, p. 1-21.

32. Jacques Ozouf, Mona Ozouf, La république des instituteurs, Paris, Gallimard-Seuil (coll. « Hautes Études »), 1992, particulièrement p. 9-32, 331-357.

33. Antoine Prost, L'enseignement en France 1800-1967, Paris, Armand Colin, 1968.

34. Jacques Ozouf, «L'enseignement impossible », Le Nouvel Observateur, lundi 16 décembre 1968, p. 42-43.

35. Voir École pratique des hautes études. Section des sciences économiques et sociales. Annuaire. Comptes rendus des cours et conférences, 1970-1971, p. 170-171; ibid., 1971-1972, p. 151 et 200-201 ; ibid., 1972-1973, p. 154-156 et 222-223 ; ibid., 1973-1974, p.182-184 ; ibid., 1974-1975, p. 216-219.

36. Guy Caplat, «Le Service d'histoire de l'éducation. Historique et missions", Histoire de l'éducation, 1, 1978, p. 3-11.

37. François Furet, Jacques Ozouf, Lire et Écrire, op. cit., t. 1, p. 7.

38. Ibid., t. 1, p. 9.

39. Ibid., t. 1, p. 12.

40. François Furet, « L'histoire quantitative... », art. cit., p. 66.

41. Même si elles sont évoquées dès le chapitre 1 du premier tome de Lire et Écrire, p. 20-25 (à propos de la validité de l' "indicateur signature »). On trouvera des éléments développés avec un souci pédagogique marqué dans une première approche de certains des résultats de l'enquête publiée par François Furet et le statisticien Wladimir Sachs, «La croissance de l'alphabétisation en France, XVIII'-XIX ${ }^{\mathrm{e}}$ siècle », Annales ESC, XXIX, 3, 1974, p. 714-737. Il n'est pas sans intérêt pour notre propos de noter que le texte en avait présenté l'année précédente à une conférence sur «L'historien et l'ordinateur », tenue à Uppsala.

42. Sur les premières utilisations de l'analyse factorielle par les historiens, sur la rupture qu'elle a représentée par rapport à la statistique « labroussienne », sur les malentendus qui l'ont souvent accompagnés quant à ce qu'il était possible d'en attendre, voir les commentaires critiques d'Alain Guerreau, «Analyses factorielles et analyses statistiques classiques: le cas des ordres mendiants dans la France médiévale ", Annales E.S.C., XXXVI, 5,1981, p. 869-912.

43. Ibid., t. 1, p. 102-115. Voir aussi Emmet Kennedy, Marie-Laurence Netter, «Les écoles primaires sous le Directoire ", Annales historiques de la Révolution française, $53^{\mathrm{e}}$ année, janvier-mars 1981, p. 3-38.

44. Voir à ce propos, Dominique Julia (dir.), «L'École normale de l'an III : bilan d'une expérience révolutionnaire ", Revue du Nord, n³17, 1996.

45. François Furet, Jacques Ozouf, Lire et Écrire, op. cit., t. 1, p. 349. 
46. Ibid., t. 1, p. 69-175, 270-324.

47. Ibid., t. 1, p. 270-327, et les remarques concernant ce chapitre intitulé « Le rôle de l'école : le verdict de l'ordinateur ", par Antoine Prost dans son compte rendu du livre, Revue française de pédagogie, 46, janvier-mars 1979, p. 73-75.

48. Ibid., t. 1, p. 281.

49. Ibid., t. 1, p. 176-228, 245-269.

50. Ibid., t. 1, p. 188-199, 340-348.

51. Ainsi pour le Nord et le Pas-de-Calais, la Marne, la Sarthe, les Côtes-du-Nord et le Finistère, et pour une série de départements méridionaux: Tarn, Hautes-Pyrénées, Gers, Hérault, Ariège, Aveyron. Voir Michel Fleury et Pierre Valmary, art. cit., p. 76.

52. Observons que le problème posé n'est pas celui des variations d'échelle au sens que cette notion a pris bien plus tard. La démarche suivie par les auteurs de Lire et Écrire relève davantage de la critique de l'ecological fallacy, mise en valeur par les sociologues et certains historiens anglosaxons, en particulier par les chercheurs de l'Université du Michigan (Ann Arbor) avec lesquels les rapport scientifiques étaient alors fort étroits, en particulier autour du projet d'une exploitation commune de la Statistique générale de la France. Cf : Lire et Écrire, t.1, p. 44 sq.

53. Pierre Lévêque, "Problèmes de l'alphabétisation en Bourgogne sous la monarchie censitaire », in F. Furet, J. Ozouf, op. cit., t. 2, p. 153-186.

54. Jean-Paul Giret, «La vie scolaire et les progrès de l'instruction populaire en Eure-et-Loir », mémoire dactylographié, Université de Tours, 1973. Voir F. Furet, J. Ozouf, op. cit., t. 1, p. 187-188. 55. André Siegfried, Tableau politique de la France de l'Ouest sous la Troisième République, Paris, Armand Colin, 1913, p. 20.

56. Muriel Jeorger, "L'alphabétisation dans l'ancien diocèse de Rouen au XVII ${ }^{\mathrm{e}}$ et XVIII ${ }^{\mathrm{e}}$ siècle », in F. Furet, J. Ozouf, op. cit., t. 2, p. 101-136; Marie-Madeleine Compère, «École et alphabétisation en Languedoc aux XVII ${ }^{\mathrm{e}}$ et XVIII ${ }^{\mathrm{e}}$ siècles ", ibid., t. 2, p. 43-99.

57. Ainsi pour le diocèse de Montpellier, dans la région de la garrigue, où les scores élevés de scolarisation correspondent à un mouvement récent de création des écoles, ibid., t. 2, p. 57 et 66.

58. Ibid., t. 2, p. 88-90, 92. Dans sa contribution sur l'alphabétisation urbaine en Aquitaine, JeanPierre Poussou ne conclut pas non plus à une avance systématique des protestants sur les catholiques, même si les résultats obtenus pour les villes d'Orthez et de La Rochelle le confirment, ibid., t. 2, p. 318-320.

59. Eugène Fontaine de Resbecq, Histoire de l'enseignement primaire avant 1789 dans les communes qui ont formé le département du Nord, Lille, L. Quarré, 1878. L'auteur fournit pour chaque commune les signatures au mariage entre 1750 et1790. Les arrondissements les plus ruraux, ceux d'Avesnes et de Dunkerque, sont aussi les plus alphabétisés.

60. François Furet, Jacques Ozouf, op. cit., t. 1, p. 231-259.

61. Michael Sanderson, "Literacy and social mobility in the industrial revolution in England", Past and Present, 56, août 1972, p. 75-104; du même auteur, "Social change and elementary education in industrial Lancashire, 1780-1840», Northern History, t. 3, 1968, p. 131-154

62. François Furet, Jacques Ozouf, op. cit., t. 2, p. 337.

63. Jean Boutier, Philippe Boutry, Serge Bonin (dir.), Atlas de la Révolution française, 6, Les sociétés politiques, Paris, Éditions EHESS, 1992.

64. Émile Guillaumin, La vie d'un simple. Mémoires d'un métayer, Paris, Nelson, 1904. Voir à ce propos, Agnès Roche, Émile Guillaumin. Un paysan en littérature, Paris, Éditions du CNRS, 2006.

65. Voir Michel Vovelle, " $\mathrm{Y}$ a-t-il eu une révolution culturelle au XVIII ${ }^{\mathrm{e}}$ siècle ? À propos de l'éducation populaire en Provence ", Revue d'histoire moderne et contemporaine, 1975, p. 89-141 ; Anne-Marie Granet-Abisset, "Entre autodidaxie et scolarisation. Les Alpes briançonnaises », Histoire de l'éducation, 70, mai 1996, p. 111-141. 
66. La vie pénible et laborieuse du colporteur Esmieu. Récit de la vie de Jean-Joseph Esmieu, Mane, Barcelonnette, Les Alpes de Lumière-Sabença de la Valeia, 2002, p. 37. Le récit lui-même date de 1823.

67. Voir à ce propos Valentin Jamerey-Duval, Mémoires. Enfance et éducation d'un paysan au XVIII siècle, présentation et notes par Jean-Marie Goulemot, Paris, Le Sycomore, 1981. Sur cet apprentissage, voir Jean Hébrard, «Comment Valentin Jamerey-Duval apprit-il à lire? L'autodidacte exemplaire ", in Pratiques de la lecture, Roger Chartier (dir.) Marseille, Rivages, 1985, p. 23-60. Voir également Pierre-Jean Grosley, La vie de Grosley écrite par lui-même, Londres-Paris, 1787, p.16: l'érudit troyen, né en 1718, a appris à lire de sa servante, elle-même analphabète : «Elle savait par cœur plusieurs morceaux de Corneille et tout Malherbe, dont ses maitres s'occupaient dans leurs

soirées d'hiver, tandis qu'au coin du feu elle filait la quenouille. [...] Quoiqu'elle ne sût point lire, c'est elle qui me l'a appris : une demi-heure dans chaque soirée était consacrée à une lecture que je faisais dans les Figures de la Bible. J'étais obligé de recommencer chaque phrase, tant qu'elle ne l'entendait point de manière à en saisir le sens, qu'elle m'amenait par là à sentir moimême. Quand je lisais sans m'arrêter aux points et aux virgules, elle frappait le livre du bout de son fuseau, en me disant d'arrêter. " Voir également le numéro Autodidaxies XVI ${ }^{e}-X X^{e}$ siècles (dir. Willem Frijhoff), Histoire de l'éducation, 70, mai 1996.

68. François Furet, Jacques Ozouf, op. cit., t. 1, p.274.

69. Voir à ce propos l'essai d'histoire comparée, proposé par Antoine Follain, Le village sous l'Ancien Régime, Paris, Fayard, 2008.

70. Voir à ce propos Jean Meyer, «Alphabétisation, lecture et écriture. Essai sur l'instruction populaire en Bretagne du XVI ${ }^{\mathrm{e}}$ au XIX siècle ", Actes du $95^{e}$ Congrès national des Sociétés savantes, Reims, 1970, section d'histoire moderne et contemporaine, t. 1, Paris, 1974, p. 333-353.

71. François Furet, Jacques Ozouf, op. cit., t.1, p. 199-229.

72. Voir Philippe Moret, «L'inspecteur et la béate. Un choc de cultures », in Un voyage au pays des béates, Auguste Rivet, Phillipe Moret, Pierre Burger, André Crémilleux, (dirs.) Romagnat, Éditions Gérard Tisserand, 2003, p. 27-66, 175-178. Le rapport cité se trouve aux AN, $F^{17} 9265$.

73. La recherche sur l'histoire des pratiques de lecture dans les périodes anciennes et sur celle des méthodes d'apprentissage a beaucoup apporté sur ce point. Voir, par exemple, Jean-Yves Seradin, "Une autre façon d'enseigner la lecture au XVIII ${ }^{\mathrm{e}}$ siècle. La méthode des mots entiers ", Les Actes de Lecture, 97, mars 2007, p. 22-35; Anne-Marie Chartier, L'école et la lecture obligatoire. Histoire et paradoxes des pratiques d'enseignement de la lecture, Paris, Retz, 2007, particulièrement p. 73-129.

74. François Furet, Jacques Ozouf, Reading and Writing: Literacy from Calvin to Jules Ferry, Cambridge-Londres-New York, Cambridge University Press, 1982.

75. On trouvera à ce propos un premier aperçu dans Marie-Madeleine Compère, L'histoire de l'éducation en Europe. Essai comparatif sur la façon dont elle s'écrit, Berne-Paris, Peter LangInstitut national de la recherche pédagogique, 1995, p.115-154; voir aussi Reiner Prass, "Signierfähigkeit und Schriftkultur. Methodische Überlegungen und neuere Studien zur Alphabetisierungsforschung in Frankreich und Deutschland ", Francia, t. 25/2, 1998, p. 175-197.

76. François Furet, Jacques Ozouf, op. cit., t. 1, p. 349-369.

77. Voir Kaspar von Greyerz, Hans Medick, Patrice Veit (dirs.), Von der dargestellten Person zum erinnerten Ich Europäische Selbstzeugnisse als historische Quellen 1500-1850, Cologne, Böhlau, 2001 ; Rudolf Dekker, Ego documents and history. Autobiographical Writing in its social context since the Middle Ages, Hilversum, Verloren, 2002 ; Louis Châtellier, Philippe Martin (dirs.), L'écriture du croyant, Turnhout, Brepols, 2005 ; Jean-Pierre Bardet, François-Joseph Ruggiu (dir.), Au plus près du secret des cœurs? Nouvelles lectures des écrits du for privé en Europe $d u X I^{e}$ au XVIII siècle, Paris, Presses de l'université de Paris-Sorbonne, 2005 ; Michel Cassan, Jean-Pierre Bardet, François-Joseph Ruggiu 
(dir.), Les écrits du for privé. Objets matériels, objets édités, Limoges, Presses universitaires de Limoges, 2007.

78. Voir par exemple Reiner Press, « Das Kreuz mit den Unterschriften. Von der Alphabetisierung zur Schriftkultur», Historische Anthropologie, t. 9, 2001, p. 384-404; Antoine Follain, Le village sous l'Ancien Régime, Paris, Fayard, 2008, p. 246-269.

\section{AUTEURS}

DOMINIQUE JULIA

EHESS/CRH/CARE,

JACQUES REVEL

EHESS/CRH 